\title{
Numerical Simulations of Particle Transport and Deposition Experiments at Gas Turbine Temperatures
}

\author{
Peter R. Forsyth*, David R.H. Gillespie ${ }^{\dagger}$, and Matthew McGilvray \\ Department of Engineering Science, University of Oxford, Oxford, OX1 3PJ
}

\begin{abstract}
The ingestion of micron-sized particulates into gas turbine engines is known to cause a number of damage mechanisms, from in-flight engine to component life-reduction. Numerical simulations are presented of particle deposition experiments at temperatures representative of gas turbine secondary air systems. The continuous random walk (CRW) model is applied to simulate the fluid turbulent fluctuations observed by the particle in a steady simulation. The CRW model assessed for the first time under an external body force, namely gravitational conditions perpendicular to the bulk flow. The introduction of the external body force is seen to require a redevelopment of the numerical integration time step. This captures the crossing trajectories effect. At ambient isothermal conditions, simulation-experimental differences were seen to be 0.9-49.7\%; at high temperature isothermal conditions the differences were seen to be 12.6-50.1\%. Simulations of non-isothermal flows were able to capture thermophoretic effects well through the use of the Beresnev and Chernyak thermophoretic coefficient, rather than the widely-used Talbot coefficient.
\end{abstract}

\section{Nomenclature}

Latin

$C_{c} \quad$ Cunningham slip correction factor

$C_{f} \quad$ Skin friction coefficient $\left(\frac{\tau_{w}}{\frac{1}{2} \rho_{g} U^{2}}\right)$

$D \quad$ Flow Diameter (m)

$d_{p} \quad$ Particle diameter $(\mathrm{m})$

$d t \quad$ Integration time step (s)

$f_{d} \quad$ Deposition fraction

$\overline{f_{d}} \quad$ Normalised deposition fraction

$g \quad$ Gravitational acceleration $\left(\mathrm{m} / \mathrm{s}^{2}\right)$

$k \quad$ Turbulent kinetic energy $\left(\mathrm{m}^{2} / \mathrm{s}^{2}\right)$

$k_{g} \quad$ Gas thermal conductivity $(\mathrm{W} / \mathrm{m} / \mathrm{K})$

$k_{p} \quad$ Particle thermal conductivity $(\mathrm{W} / \mathrm{m} / \mathrm{K})$

$K n \quad$ Knudsen number $\left(\frac{2 l_{g}}{d_{p}}\right)$

$L \quad$ Length over which deposition occurs (m)

$l_{g} \quad$ Gas mean free path (m)

Re Reynolds number $\left(\frac{\rho_{f} \overline{U_{g}} D}{\mu_{g}}\right)$

$R e_{p} \quad$ Particle Reynolds number $\left(\frac{\rho_{g} V_{\text {rel }} d_{p}}{\mu_{g}}\right)$

Stk Stokes number $\left(\frac{C_{c} \rho_{p} \rho_{f} d_{p}^{2}}{18 \mu_{g}} \frac{D}{\overline{U_{g}}}\right)$

$T \quad$ Temperature (K)

$\overline{U_{g}} \quad$ Mean gas velocity $(\mathrm{m} / \mathrm{s})$

$u^{*} \quad$ Friction velocity $(\mathrm{m} / \mathrm{s})$

$V_{\text {rel }} \quad$ Relative particle-gas velocity $(\mathrm{m} / \mathrm{s})$

$V_{d}^{+} \quad$ Non-dimensional deposition velocity $\mathbf{x} \quad$ Particle position (m)

$y^{+} \quad$ Non-dimensional wall distance $\left(\frac{\rho_{f} u^{*} y}{\mu_{g}}\right)$

Greek

$\beta \quad$ Ratio of Lagrangian to Eulerian time scales

$\Lambda \quad$ Thermal conductivity ratio $\left(k_{p} / k_{g}\right)$

$\mu \quad$ Dynamic viscosity (Pa.s)

$\Phi \quad$ Thermophoretic coefficient

$\rho \quad$ Density $\left(\mathrm{kg} / \mathrm{m}^{3}\right)$

$\sigma \quad$ Root mean squares fluctuating velocity $(\mathrm{m} / \mathrm{s})$

$\tau_{L} \quad$ Lagrangian time scale (s)

$\tau_{p} \quad$ Particle relaxation time (s)

$\tau_{p}^{+} \quad$ Non-dimensional particle relaxation time

Scripts

d Deposition

D Drag

G Gravitational

g Gas

p Particle

in Inlet

out Outlet

Th Thermophoretic

* Postdoctoral Research Assistant in Engineering Science

$\dagger$ Professor of Engineering Science

†Professor of Engineering Science 


$\begin{array}{llll}w & \text { Wall } & \text { DRW } & \text { Discrete random walk } \\ + & \text { Non-dimensional } & \text { EIM } & \text { Eddy interaction model } \\ & \text { LPT } & \text { Lagrangian particle tracking } \\ \text { Abbreviations } & \text { RANS } & \text { Reynolds-averaged Navier-Stokes } \\ \text { CRW Continuous random walk } & \text { UDF } & \text { User defined function }\end{array}$

\section{Introduction}

$\mathrm{T}$

HE expansion of civil aviation within regions containing high levels of airborne particulates and flights through volcanic ash clouds [1], has led to greater interest in the motion and deposition of micron-sized particulates at gas turbine engine relevant conditions. Particulates, including sands, salts, and volcanic ashes, can be ingested into the engine [2]. Particle effects in the main gas path include erosion and fouling of compressor blades [3], and deposition onto nozzle guide vanes and high pressure turbine blades [4]. Particulates small enough to enter the secondary air systems $(<\sim 10 \mu \mathrm{m})$ have been seen to cause blockage of internal cooling features, reducing cooling effectiveness [5]. Particles of this size can be strongly influenced by the turbulent fluctuations of the gas, which have been shown to increase the rate of particulate deposition by orders of magnitude [6].

Numerical simulation of particle-laden flows is largely done using two approaches. The first, Eulerian, models the solid particle phase as a second fluid phase, solving similar equations to the continuous phase. Whilst good for dense particle suspensions, Eulerian particle tracking suffers difficulties with particle-wall interactions, and in flows with a low volumetric ratio of particles to fluid.

Lagrangian particle tracking (LPT) is widely applied to complex geometries, where large numbers of particles are injected into the domain and tracked individually. The number of particles should be increased until stationary deposition statistics are achieved. A one-way coupling is generally assumed, implying that the particle-fluid volumetric ratio is less than $10^{-6}$. Particles are tracked by integrating their equation of motion,

$$
\frac{d^{2} \mathbf{x}}{d t^{2}}=\frac{1}{\tau_{p}} \frac{C_{D} R e_{p}}{24} \mathbf{V}_{r e l}+\mathbf{a}_{G}+\mathbf{a}_{T h}
$$

where the three terms on the right hand side are the contributions from drag, gravity, and thermophoresis. The drag coefficient $C_{D}$ is calculated from the following expressions:

$$
C_{D}= \begin{cases}\frac{24}{R e_{p}} & \text { for } \operatorname{Re}_{p}<1, \\ \frac{24}{R e_{p}}\left(1+0.15 R e_{p}^{0.687}\right) & \text { for } 1 \leq R e_{p}<400 \text { [7]. }\end{cases}
$$

The particle acceleration due to gravity is well-approximated by $\mathbf{a}_{G}=\mathbf{g}$ for flows where $\rho_{p} \gg \rho_{g}$. The introduction of a temperature gradient to the gas causes particles to experience a thermophoretic acceleration in the negative direction of the temperature gradient, proportional to its magnitude,

$$
\mathbf{a}_{T h}=-\Phi \frac{\mu_{g}^{2} d_{p}}{2 \rho_{g} m_{p}} \frac{\nabla T_{g}}{T_{g}},
$$

where $\boldsymbol{\nabla} T_{g}$ is the gas temperature gradient, and $\Phi$ the thermophoretic coefficient. The thermophoretic coefficient is a function of thermal conductivity ratio, $\Lambda$, and Knudsen number, $K n$. Hence, for $T_{g}>T_{w}$, the rate of deposition observed is increased in comparison to isothermal conditions ('increasing' thermophoresis); for $T_{g}<T_{w}$, the reverse is observed ('decreasing' thermophoresis).

\begin{tabular}{cc}
\hline \hline Author & Expression \\
\hline \hline Brock [8] (Talbot et al. [9]) & $\Phi=\frac{12 \pi K_{t c}\left(1+C_{e} \Lambda K n\right)}{\left(1+3 C_{m} K n\right)\left(2+\Lambda+2 C_{e} \Lambda K n\right)}$ \\
Beresnev and Chernyak [10] & $\Phi=\frac{2 \pi}{K n}\left[\frac{f_{11}+\Lambda f_{21}}{f_{33}+(1+2.5 \Lambda K n) f_{41}}\right]$ \\
Young [11] & $\Phi=\frac{12 \pi\left[K_{t c}\left(1+\Lambda C_{e} K n\right)+3 C_{m} K n\left(1-\Lambda+\Lambda C_{e} K n\right)\right]}{\left[1+3 K n \exp \left(-C_{i n t} / K n\right)\right]\left(1+3 C_{m} K n\right)\left(2+\Lambda+2 \Lambda C_{e} K n\right)}$ \\
\hline \hline
\end{tabular}

Table 1 Expressions for the thermophoretic coefficient $\Phi$ 
A number of expressions for the thermophoretic coefficient have been published, three are shown in Table 1 The coefficient proposed by Brock [8] was adjusted by Talbot et al. [9] to make the expression applicable to all flow regimes. Beresnev and Chernyak [10] developed an analysis based on the S-model, a higher-order linearisation of the Boltzmann equation collision integral than the (more widely used) BGK equation. The formulation for thermophoretic coefficient $\Phi$ is dependent on a number of functions, $f_{k, 1}$, for $k=1-4$, tabulated in their paper, requiring interpolation for practical use. The analytic expression of Young [11] addressed this, producing an interpolation that linked a low-Kn expression based on the Grad 13-moment approach with the Waldmann [12] free molecular limit. The Talbot et al. [9] formulation is extremely widely used, despite evidence that it over-predicts the thermophoretic force for high particle-gas thermal conductivity ratio combinations.

For internal flows the non-dimensional particle relaxation time, $\tau_{p}^{+}$, is a useful description of a particle's response to the local flow conditions. This is the ratio of the particle relaxation time $\left(\tau_{p}=C_{c} \rho_{p} d_{p}^{2} / 18 \mu\right)$ to the gas wall time scale $\left(\tau_{g, w}=\mu / \rho\left(u^{*}\right)^{2}\right)$,

$$
\tau_{p}^{+}=S t k \cdot R e \cdot C_{f}=\frac{C_{c} \rho_{g} \rho_{p} d_{p}^{2}\left(u^{*}\right)^{2}}{18 \mu^{2}},
$$

The deposition rate is frequently defined by its non-dimensional deposition velocity $V_{d}^{+}$, a non-dimensional volumetric deposition rate per unit area of surface,

$$
V_{d}^{+}=\frac{V_{d}}{u^{*}}=\frac{1}{4} \frac{D}{L} \frac{\overline{U_{g}}}{u^{*}} \ln \left(\frac{1}{1-f_{d}}\right),
$$

From the work of [6], amongst others, it is known that the deposition of particles in the range $0.3<\tau_{p}^{+}<20$ from turbulent flows is strongly affected by fluid turbulent fluctuations. In order to attempt to capture the stochastic effect of fluid turbulence on particle motion in RANS simulations, the eddy interaction model/discrete random walk model (EIM/DRW) [13] has been frequently employed. This has been shown however to have a number of deficiencies for the modelling of particle motion in anisotropic turbulence [14], causing spurious deposition results, up to $100 \times$ above experimentally-measured values. The continuous random walk (CRW) model [15], provides a more physically realistic description of the turbulent gas fluctuations seen by suspended particles for wall-bounded flows. The CRW solves the normalised Langevin equation, splitting the flow into two regions: the isotropic bulk, and the anisotropic boundary layer. The CRW model has been applied to a number of geometries, including pipe, bend [15], thoracic [16], and mixing junction [17] geometries.

Whilst much of the fundamental experimental work on particle deposition has been carried out on vertical flows (for example [6]), the introduction of external body forces, e.g., gravity, have been shown to significantly alter deposition trends. For horizontal pipe flow, [18, 19], increases in $V_{d}^{+}$of 10-100x have been seen, relative to matching $\tau_{p}^{+}$values in vertical flow conditions.

This paper addresses the simulation of horizontal flows under gravitational conditions at ambient and high temperatures using the CRW model. A new integration time step is developed. The simulation results using the new integration time step are presented for isothermal ambient and high temperature conditions, indicating improved matching to the experimental data., and also non-isothermal/thermophoretic high temperature conditions. Comparisons of simulations using different thermophoretic coefficient formulations shows that predictions using the Beresnev and Chernyak formulation are up to an order of magnitude closer to the experimental data than those using the Talbot formulation.

\section{Experiments simulated}

The experiments simulated in this current study are fully described in Forsyth et al. [20]. The authors deposited 2.0-6.5 $\mu \mathrm{m}$ particles of $\mathrm{NaCl}$ from horizontal turbulent pipe flow, at gas temperatures $20-480^{\circ} \mathrm{C}$, and metal temperatures 20-730 ${ }^{\circ} \mathrm{C}$. Bulk Reynolds numbers were $6,500-27,000$, calculated from

$$
R e=\frac{4 \dot{m}}{\pi \mu_{T_{g}} D},
$$

where viscosity is calculated based on test section centreline gas temperature $T_{g 4}(10 \mathrm{~mm}$ downstream of test section, see Fig. 2), and the mass flow rate measured using a mass flow controller. 


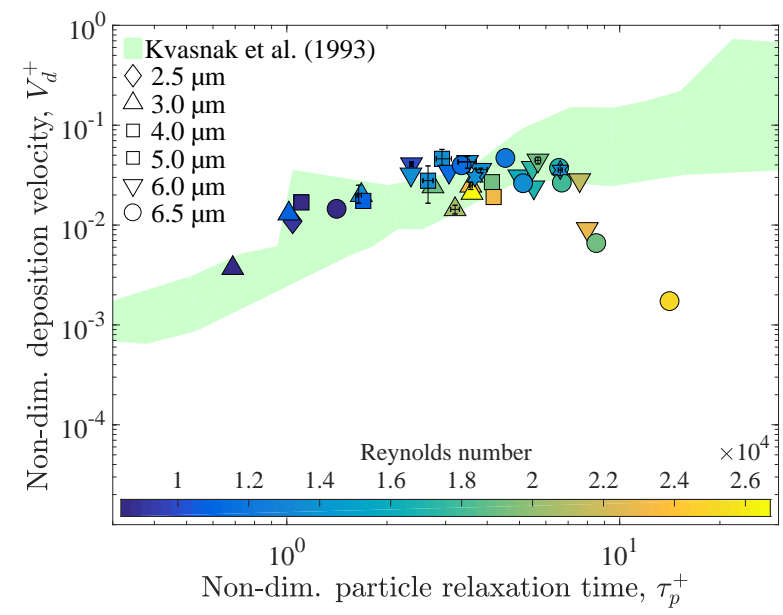

(a) Ambient, isothermal

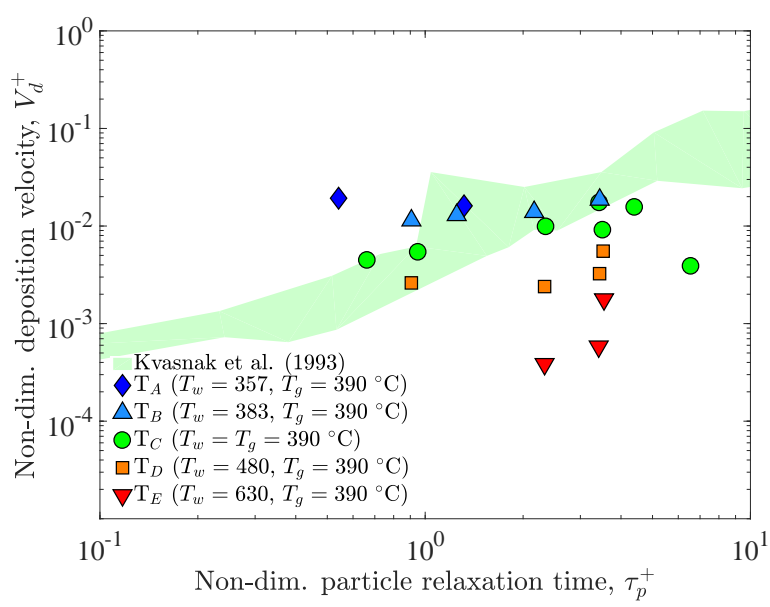

(b) High temperature, thermophoretic

Fig. 1 Experimental results from Forsyth et al. [20] (plot markers), and Kvasnak et al. [21] (green regions indicating spread of data)

Under isothermal conditions, the ambient, Fig. 1a and high temperature deposition trends were similar. For $\tau_{p}^{+}<8$, $V_{d}^{+}$rises with increasing $\tau_{p}^{+}$. For $\tau_{p}^{+} \geq 8$, a reduction in deposition was seen for the highest kinetic energy particles, thought to be a rebound or removal effect. Error bars indicate the spread of data from repeated tests was small; a mean of $14 \%$, with a standard deviation of $11 \%$. The maximum deviation was $40 \%$.

Under thermophoretic (non-isothermal) conditions, Fig. $1 \mathrm{~b}$, deposition increased by factors of up to 4.8 (blue diamonds and triangles) for $T_{g}>T_{w}$, and decreased by factors of up to 560 (orange squares, red triangles) for $T_{g}<T_{w}$. The strength of the change was seen to be highly dependent on the direction and magnitude of temperature gradient, and the particle size. For the strongest decreasing thermophoretic conditions (labelled $\mathrm{T}_{E}$ ), deposition was only noted on the bottom surface of the passage, indicating that gravitational effects were present.

\section{Numerical set-up}

\section{A. Numerical domain}

The geometry and boundary conditions for the numerical simulations match those used experimentally, Fig. 2 . $\mathrm{I}_{1}$, $\mathrm{I}_{2}$ : Bulk and atomiser inlets respectively, $\dot{m}_{g 1}, T_{g 1}$ as measured in experiment; $\mathrm{O}_{1}$ : Outlet, pressure outlet to $0 \mathrm{~Pa}$ as experiment; $\mathrm{W}_{1}, \mathrm{~W}_{3}$ : Bulk and atomiser tubes respectively; adjusted to give $T_{g 3}, T_{g 4}$ as measured; $\mathrm{W}_{2}$ : Atomiser tube, ambient $(295 \mathrm{~K})$ as experiment; $\mathrm{W}_{3}$ : Mixing chamber; adjusted to give $T_{g 4}$ as experiment; $\mathrm{W}_{4}$ : Tube, insulated as experiment; $\mathrm{W}_{5}, \mathrm{~W}_{6}$ : Oven and test section respectively, matched to mean of $T_{w 1-3}$ as experiment. The centre of the atomiser tube enters the mixing chamber at an axial distance of $303 \mathrm{~mm}$ from bulk inlet $\mathrm{I}_{1}$. Boundary condition values are given in Table 2. 'Thermophoretic condition' refers to the wall-gas temperature pairings used for thermophoretic simulations.

The particle size distribution was measured before each test using an optical particle sizer (OPS). Particles are binned by observed diameter, using a laser scattering technique. Distributions by number for nominal particle sizes 2.5-6.0 $\mu \mathrm{m}$ are shown in Table 3 The mean particle size for each bin is used in reconstructing the distributions simulated, assuming a uniform particle density $\left(\rho=2000 \mathrm{~kg} / \mathrm{m}^{3}\right)$. Particles were injected into the domain in the atomiser tube, from a plane parallel to $\mathrm{I}_{2}$, placed $40 \mathrm{~mm}$ above the main passage axis in the y-direction. The injection plane was a uniformly spaced grid of points. 7600 particles were injected for each particle diameter, unless fewer than 50 were deemed to have stuck, in which case 31000 particles were injected. The particle boundary condition was lossless rebound in the atomiser tube, and 'all-stick' on all other appropriate surfaces $\left(\mathrm{W}_{3-6}\right)$. Under the 'all-stick' boundary condition, if the centre of the particle is found to come within one particle radius of the wall, it is assumed stuck.

The realisable $k-\epsilon$ turbulence model with enhanced wall treatment was used for closure of the Reynolds-averaged Navier-Stokes equations. Trials with the Reynolds stress model (RSM) did not indicate significant differences to particle statistics. Spatial discretisation of scalars was carried out using the second-order upwind scheme. The 'coupled' 


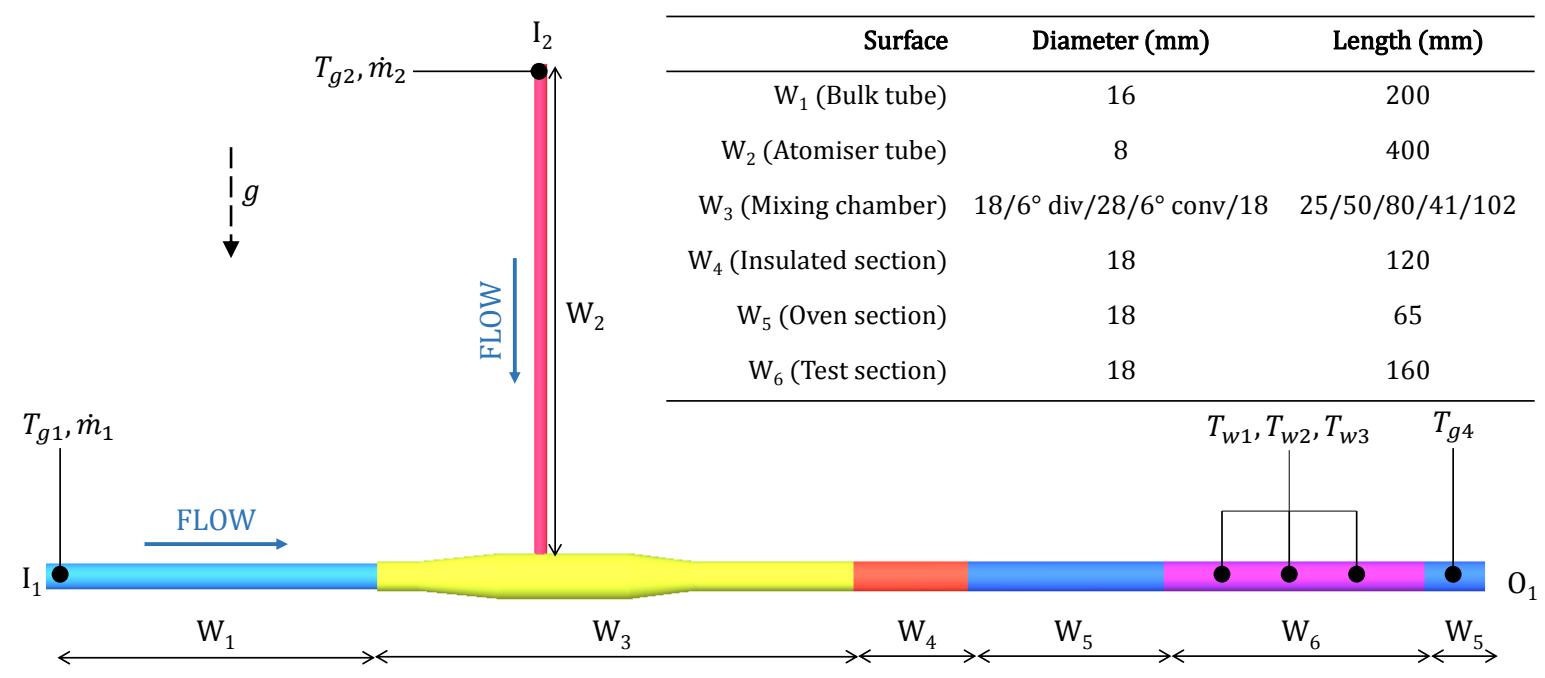

Fig. 2 Numerical domain for simulations of experimental work, indicating boundary condition measurement locations. Gravity included in the vertical direction. Arrows indicate flow direction. Boundary condition values reported in Table 2

pressure-velocity coupling scheme was used for its efficiency and Green-Gauss Node-Based gradient discretisation. Gas density was calculated from the ideal gas law. Gas viscosity was calculated by Sutherland's law. Gas specific heat capacity [22] and thermal conductivity [23] were correlated against gas temperature (K),

$$
\begin{gathered}
c_{p}=716.8+0.812 T_{g}-5.334 \times 10^{-5} T_{g}^{2}+1.445 \times 10^{-7} T_{g}^{3}, \\
k=0.0169+5.113 \times 10^{-5} T_{g} .
\end{gathered}
$$

Single precision calculations were carried out. Scaled residuals were of the order of $10^{-5}$ for continuity, $10^{-6}$ for turbulence properties, and $10^{-7}$ for energy and velocities. Maximum net mass flow rate was of the order of $10^{-7} \%$ of inlet mass flow rate. The gravitational force was switched on. The Lagrangian particle tracking used the drag, gravitational, and thermophoretic particle forces.

A grid independence study was carried out. Four meshes were generated, coarse: 1.74, medium: 2.86, fine: 6.08, very fine: $8.74 \mathrm{M}$ cells. Mean test section pressure, net convective heat flux into the domain through walls, and particle deposition rate were assessed. These parameters showed the medium density grid ( $2.86 \mathrm{M}$ cells, 10 prism layers, first cell height $0.03 \mathrm{~mm}$, expansion ratio $1.25, y^{+}<1$ across domain) was enough to generate a grid-independent solution. For particle tracking, $7600-69000$ particles were injected, depending on particle diameter. Deposition was seen to converge for all meshes for the larger particles $(3-6 \mu \mathrm{m})$, and within a factor of two between the medium and fine meshes. This convergence is considered acceptable in relation to the spread of published data.

\section{B. Continuous random walk model}

The CRW model of Dehbi [15] is another model for the instantaneous fluid fluctuating velocity as seen by a particle, based upon the normalised Langevin equation [24]. Full details of the model are given in [15]; the below provides an overview. The general form of the increment in normalised fluctuating velocity is given by

$$
d\left(\frac{u_{i}}{\sigma_{i}}\right)=-\left(\frac{u_{i}}{\sigma_{i}}\right) \frac{d t}{\tau_{L}}+\sqrt{\frac{2}{\tau_{L}}} d \xi_{i}+A_{i} d t .
$$

Here $\sigma$ is root mean square fluctuating velocity, $\tau_{L}$ the Lagrangian (integral) time scale, $d \xi$ a Gaussian random number with zero mean and variance $d t$, therefore is solved as an incremental Weiner process, $d \xi=\xi \sqrt{d t}$ where $\xi$ is a Gaussian random number, with zero mean and variance $d t$, and $A_{i}$ the drift correction, which prevents non-physical drift into, and accumulation within, the boundary layer. 


\begin{tabular}{|c|c|c|c|c|c|c|c|c|c|c|c|c|}
\hline $\operatorname{Re}$ & $\begin{array}{c}\text { Thermophoretic } \\
\text { condition }\end{array}$ & $\begin{array}{l}\dot{m}_{1} \\
\mathrm{~g} / \mathrm{s}\end{array}$ & $\begin{array}{c}T_{g 1} \\
\mathrm{~K}\end{array}$ & $\begin{array}{l}\dot{m}_{2} \\
\mathrm{~g} / \mathrm{s}\end{array}$ & $\begin{array}{c}T_{g 2} \\
\mathrm{~K}\end{array}$ & $\begin{array}{c}T_{w 1} \\
\mathrm{~K}\end{array}$ & $\begin{array}{c}T_{w 2} \\
\mathrm{~K}\end{array}$ & $\begin{array}{c}T_{w 3} \\
\mathrm{~K}\end{array}$ & $\begin{array}{c}T_{w 4} \\
\mathrm{~K}\end{array}$ & $\begin{array}{c}T_{w 5} \\
\mathrm{~K}\end{array}$ & $\begin{array}{c}T_{w 6} \\
\mathrm{~K}\end{array}$ & $\begin{array}{c}T_{g 4} \\
\mathrm{~K}\end{array}$ \\
\hline \multicolumn{13}{|c|}{ Ambient, isothermal } \\
\hline 10700 & - & 1.42 & 300 & 1.34 & 300 & 300 & 300 & 300 & 300 & 300 & 300 & 300 \\
\hline 14000 & - & 2.37 & 300 & 1.18 & 300 & 300 & 300 & 300 & 300 & 300 & 300 & 300 \\
\hline 19000 & - & 3.33 & 300 & 1.60 & 300 & 300 & 300 & 300 & 300 & 300 & 300 & 300 \\
\hline 23000 & - & 3.85 & 300 & 2.07 & 300 & 300 & 300 & 300 & 300 & 300 & 300 & 300 \\
\hline \multicolumn{13}{|c|}{ High temperature, isothermal } \\
\hline 6500 & - & 1.98 & 627 & 0.98 & 295 & 900 & 295 & 900 & $\dot{Q}=0$ & 663 & 663 & 661 \\
\hline 7800 & $\mathrm{~T}_{C}$ & 2.37 & 670 & 1.18 & 295 & 880 & 295 & 880 & $\dot{Q}=0$ & 663 & 663 & 663 \\
\hline 10000 & - & 3.45 & 643 & 1.11 & 295 & 860 & 295 & 860 & $\dot{Q}=0$ & 659 & 659 & 662 \\
\hline \multicolumn{13}{|c|}{ High temperature, thermophoretic (non-isothermal) } \\
\hline \multirow{4}{*}{7800} & $\mathrm{~T}_{A}$ & 2.37 & 718 & 1.18 & 295 & 900 & 295 & 845 & $\dot{Q}=0$ & 630 & 630 & 659 \\
\hline & $\mathrm{T}_{B}$ & 2.37 & 679 & 1.18 & 295 & 900 & 295 & 870 & $\dot{Q}=0$ & 656 & 656 & 663 \\
\hline & $\mathrm{T}_{D}$ & 2.36 & 595 & 1.19 & 295 & 950 & 295 & 880 & $\dot{Q}=0$ & 753 & 753 & 666 \\
\hline & $\mathrm{T}_{E}$ & 2.58 & 518 & 0.97 & 295 & 780 & 295 & 867 & $\dot{Q}=0$ & 903 & 903 & 664 \\
\hline
\end{tabular}

Table 2 Boundary conditions from experiment as used in simulations reported in this paper. Nominal thermal conditions defined by test section temperature, $T_{\mathrm{w} 6}$, and test section gas temperature, $\mathbf{T}_{\mathrm{g} 4}$.

\begin{tabular}{lccccccc}
\hline \hline Nominal & \multicolumn{8}{c}{ Mean particle bin size $(\mu \mathrm{m})$} \\
$d_{p}(\mu \mathrm{m})$ & 2.04 & 2.47 & 3.05 & 3.67 & 4.49 & 5.49 & 6.79 \\
\hline \hline 2.5 & 0.19 & 0.48 & 0.15 & 0.10 & 0.06 & 0.02 & 0.00 \\
3.0 & 0.01 & 0.01 & 0.35 & 0.38 & 0.18 & 0.06 & 0.01 \\
4.0 & 0.10 & 0.08 & 0.08 & 0.011 & 0.42 & 0.14 & 0.06 \\
5.0 & 0.02 & 0.02 & 0.03 & 0.04 & 0.43 & 0.29 & 0.17 \\
6.0 & 0.05 & 0.06 & 0.08 & 0.09 & 0.09 & 0.42 & 0.22 \\
\hline \hline
\end{tabular}

Table 3 Fractional particle size distribution by number from experiments as applied in simulations. Note that the $2.5 \mu \mathrm{m}$ nominal particle size was only used in the high temperature experiments and simulations. 
The model is calculated in two layers, the bulk and boundary layer regions, split at $y^{+}=100$. The root mean square fluctuating velocity $\sigma$ is defined differently for each layer. In the bulk region, $y^{+} \geq 100$, it is isotropic and calculated from the turbulent kinetic energy $k$,

$$
\sigma=\sqrt{\frac{2}{3} k}
$$

In the boundary layer, turbulence is strongly anisotropic. The model accounts for this anisotropy by imposing normalised fluid velocity fluctuations from direct numerical simulations (DNS) of channel flow. A body fitted co-ordinate system (BFCS) is applied in the streamwise (index $i=1)$, wall-normal $(i=2)$, and spanwise $(i=3)$ directions. The DNS normalised fluid velocity fluctuations in each direction are correlated against $y^{+}$,

$$
\begin{gathered}
\sigma_{1}^{+} \equiv \frac{\sigma_{1}}{u^{*}}=\frac{0.40 y^{+}}{1+0.0239\left(y^{+}\right)^{1.496}}, \\
\sigma_{2}^{+} \equiv \frac{\sigma_{2}}{u^{*}}=\frac{0.0116\left(y^{+}\right)^{2}}{1+0.203 y^{+}+0.0014\left(y^{+}\right)^{2.421}}, \\
\sigma_{3}^{+} \equiv \frac{\sigma_{3}}{u^{*}}=\frac{0.19 y^{+}}{1+0.0361\left(y^{+}\right)^{1.322}} .
\end{gathered}
$$

The Lagrangian integral time scale $\tau_{L}$ is a spatially-varying property of the flow turbulence, which characterises large-scale turbulent motion. The Lagrangian integral time scale was assumed isotropic by Dehbi, following the DNS study of Bocksell and Loth [25]. This is discussed further in Section A

The drift correction $A_{i}$ proportional to the spatial gradient of fluctuating velocity, and is shown to have a Stokes number-dependency. In the bulk region, the drift correction is given by

$$
A_{i}=\frac{1}{3 \sigma} \frac{\partial k}{\partial x_{i}} \frac{1}{1+S t k} .
$$

In the boundary layer the drift correction is set to zero in the $i=1,3$ directions, and is calculated from the DNS root mean square fluctuation in the wall-normal direction $(i=2)$,

$$
A_{2}=\frac{\partial \sigma_{2}}{\partial x_{2}} \frac{1}{1+S t k} .
$$

The initial implementation of the CRW model was provided by Ghahramani et al. [16] as a user defined functions (UDF) for Fluent, which was verified against the original [16]. Further assessment was carried out by Forsyth et al. [26], including the development of a new integration time step, $d t^{*}$,

$$
d t^{*}=\frac{\tau_{L}}{\sqrt[4]{R e}} .
$$

Application of the CRW model with Eq. 16 to simulations vertical pipe flow [26] were highly successful, Fig. 3a Close reproduction of the experimental results was seen. When this set-up was applied to initial simulations of the experimental work described in Section $\Pi$ it was found that the model was not capturing the change in deposition trend induced by the external body force, Fig $3 \mathrm{~b}$. Under these horizontal (gravitational) conditions, the predictions of the CRW model are similar to those produced for vertical flows, where gravitational effects are not present. Despite the gravitational force being activated for these horizontal simulations, it is clear that the effect of the interaction between turbophoresis and gravity on particle motion is not being captured by the CRW model in this form.

Yudine [27] introduced the concept of crossing trajectories, where external forces alter the time scales associated with particle-turbulence interaction. The Lagrangian integral time scales have also been shown to become anisotropic under gravitational conditions. The crossing trajectories effect is now included into the CRW model via adjustment of the Lagrangian time scales.

Anisotropic non-dimensional time scales were implemented in the boundary layer region from published DNS data [28]. The data were linearised over two regions $\left(y^{+} \leq 10, y^{+}>10\right)$. These are shown in Fig. 4, along with the nominally isotropic fit of Kallio and Reeks [29].

Csanady [30] produced expressions for the decorrelation time scales, relating the Lagrangian integral time scale in the wall-normal (22) direction, $\tau_{L 22}^{G}$, to the isotropic value, $\tau_{L}$, 


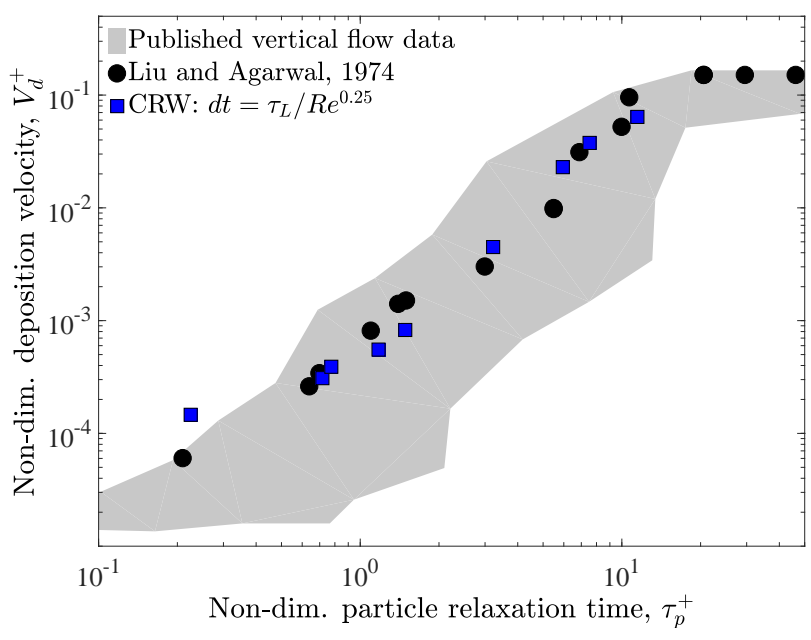

(a)

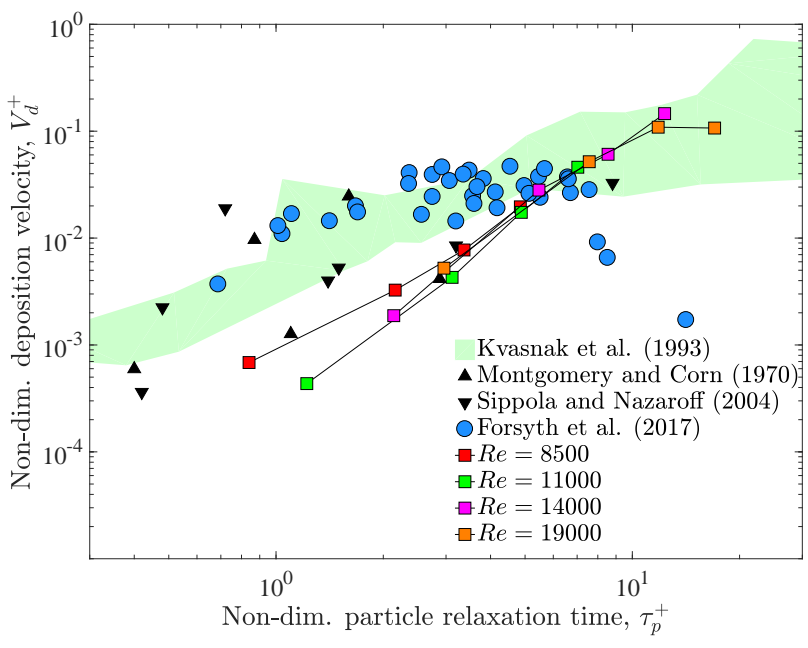

(b)

Fig. 3 Pipe flow simulations, using CRW model with initial time step dt*. (a) Vertical pipe flow simulations (adapted from [26]), showing close reproduction of the Liu and Agarwal [6] vertical experimental data. (b) Horizontal pipe flow simulations (ambient, isothermal) against horizontal deposition experiments [18-21].

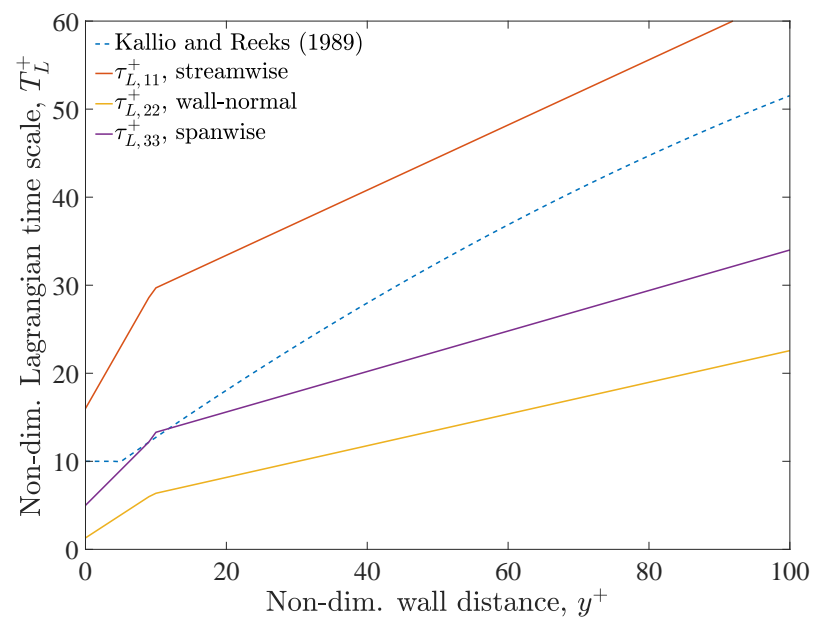

Fig. 4 Anisotropy of non-dimensional Lagrangian time scales. Directions: 1. streamwise, 2. wall-normal, 3. spanwise.

$$
\frac{\tau_{L 22}^{G}}{\tau_{L}}=\frac{1}{\sqrt{1+\left(\beta \frac{\left|\mathbf{V}_{\text {slip }}\right|}{\sigma_{g}}\right)^{2}}} .
$$

Values for $\beta$, the ratio of Lagrangian to Eulerian time scale, in the literature vary in the region 0.2-4; for these simulations in this paper we set $\beta=1.0$. The relative particle slip or drift velocity in the direction of gravity, $V_{\text {slip }}$, is calculated from the particle's terminal velocity, equating the gravitational force on a particle with Stokes (viscous) drag:

$$
\mathbf{V}_{\text {slip }}=\tau_{p} \mathbf{g} .
$$

The same result can be found by equating the gravitational force on the particle with aerodynamic drag, and applying Eq. $2 \sigma_{g}$ is the root mean square fluid velocity fluctuation in the direction of gravity. Outside the boundary layer, $\sigma_{g} \gg\left|\mathbf{V}_{\text {slip }}\right|$, hence the correction factor from Eq. 17 is not applied. 


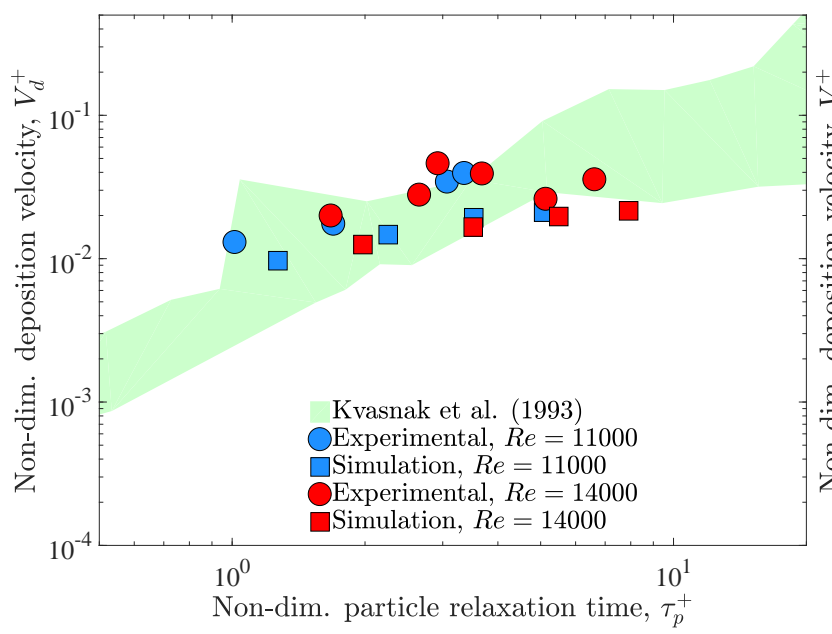

(a) $R e=11000,14000$

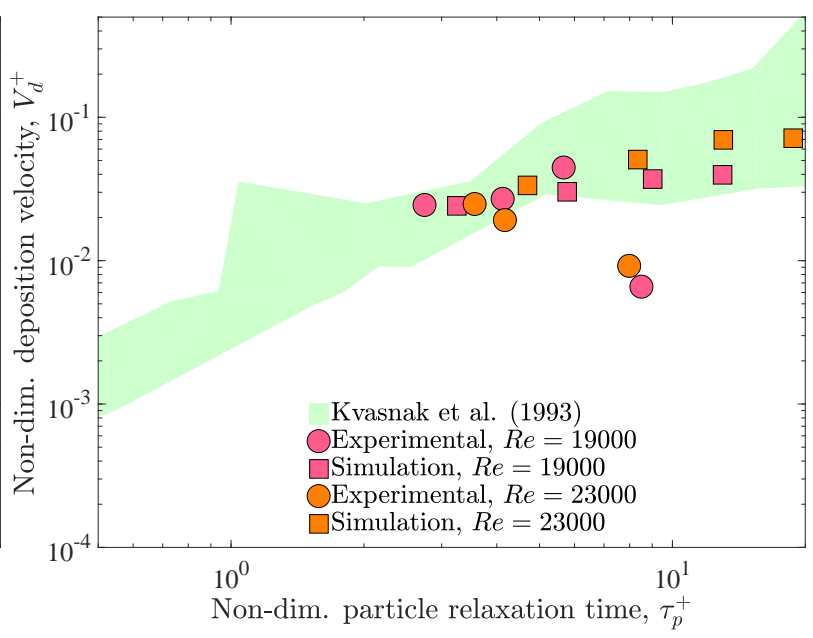

(b) $R e=19000,23000$

Fig. 5 Simulation vs. experimental data for ambient isothermal horizontal pipe flow at constant Re. Nominal particle diameters of 3.0, 4.0, 5.0, $6.0 \mu \mathrm{m}$ were simulated for each case as per Table 3 .

The wall-normal Lagrangian time scale $\tau_{L 22}^{G}$ is then substituted for the isotropic Lagrangian time scale in Eq. 16 to calculate the integration time step,

$$
d t_{L 22}=\frac{\tau_{L, 22}^{G}}{\sqrt[4]{R e}}
$$

Simulations using this time step are presented in the following section for ambient and high temperature conditions.

\section{Results}

\section{A. Ambient: Isothermal}

Isothermal simulations at ambient temperatures using Eq. 19 are presented in Fig. 5 against the experimental data [26]. Results are shown for the 3.0-6.0 $\mu \mathrm{m}$ nominal particle diameters, Table 3, at constant $R e$. As the particle size distribution does not vary with $R e$ in the simulations, and not all nominal particle sizes were obtained experimentally, as well as some extra particle sizes being run experimentally, some differences in $\tau_{p}^{+}$are seen.

Qualitative comparisons to Fig. 3bindicate that the updated time step accounting for crossing trajectory effects due to gravity has improved the simulations. For $R e=11000$, Fig. 5a, the simulations under-predicted deposition by $14.3-46.4 \%$, performing best for the smaller particle sizes $(3.0 \& 4.0 \mu \mathrm{m})$. In comparison to many published experimental datasets, for example see Fig 3 where the spread of $V_{d}^{+}$at given $\tau_{p}^{+}$can exceed two orders of magnitude. For $R e=14000$, Fig. 5a the simulations under-predicted deposition by 17.2-49.7\%. Over the range of $\tau_{p}^{+}$assessed, the rise in $V_{d}^{+}$was well simulated: $1.78 \times$ in comparison to $1.72 \times$ (experimental).

The $R e=19000$ simulations, Fig. 5b, successfully simulated the smaller particles, nominal particle diameters 3,4 , $\& \mu \mathrm{m}: 0.9,12.6,16.5 \%$ below the experimental values respectively. The $6 \mu \mathrm{m}$ particle was not well simulated, being $605 \%$ higher than the experimental value. The reduction seen in $V_{d}^{+}$for $\tau_{p}^{+}>8$ experimentally was thought to be a particle rebound or removal effect at impact [20], which the all-stick boundary condition is not attempting to model.

The $R e=23000$ simulations, Fig. 5b show significant differences to the experimental data. At the smallest nominal particle size, $d_{p}=3.0 \mu \mathrm{m}$, the simulation shows $V_{d}^{+} 35.5 \%$ higher than the experimental value. As particle size and $\tau_{p}^{+}$ increase, the difference widens to $570 \%$. As for the $R e=19000$ case, this is thought to be related to the un-modelled rebound/removal mechanism. 


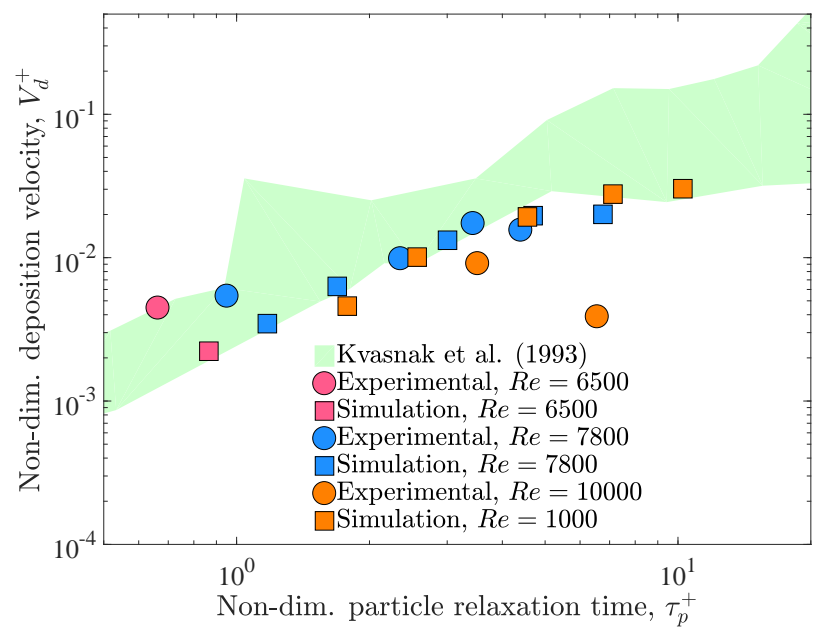

Fig. 6 Simulation vs. experimental data for high temperature isothermal pipe flow. Conditions: $\mathrm{T}_{\mathrm{g} 4}=663 \mathrm{~K}$, $\operatorname{Re}=6500-10000, d_{p}=2.5,3.0,4.0,5.0,6.0 \mu m$.

\section{B. High temperature: Isothermal}

Simulations of the high temperature isothermal experimental data were undertaken. The thermophoretic force was switched on, and the thermophoretic coefficient of Beresnev and Chernyak [10] (see following section) used, as a radial thermal gradient exists in parts of the domain. The same particle size distributions as above were used to form the nominal particle sizes $2.5-6.0 \mu \mathrm{m}$.

Deposition results are shown in Fig. 6. At the lowest Reynolds number, $R e=6500$, a single experimental value is available for comparison. Here the simulation under-predicts the experimental value by $50.1 \%$. At $R e=7800$ the simulations are closer to the experimental data, well-predicting the trend. Simulation to experimental $V_{d}^{+}$differences ranged 12.6-35.9\%. The $R e=10000$ simulations were not able to capture the reduction in $V_{d}^{+}$seen experimentally, see previous section, over-predicting observed deposition by $211-780 \%$.

\section{High temperature: Non-isothermal/thermophoretic}

Thermophoretic simulations are presented as normalised deposition fraction, $\overline{f_{d}}$, the ratio of deposition fraction under thermophoretic conditions to isothermal deposition fraction,

$$
\overline{f_{d}}=\frac{f_{d, \text { thermophoretic }}}{f_{d, \text { isothermal }}} .
$$

Hence $\overline{f_{d}}>1$ indicates that the thermophoretic conditions are increasing the rate of particle deposition; $\overline{f_{d}}<1$ indicates the reverse. The only parameter changed between each test in these cases is wall temperature; $\dot{m}_{g}(R e), d_{p}, T_{g}$ remain constant.

The three expressions for thermophoretic coefficient $\Phi$ listed in Table 1 were used for calculating the thermophoretic force on the particle, Fig. 7. A constant value of particle thermal conductivity, $4.85 \mathrm{~W} / \mathrm{m} / \mathrm{K}$ was applied. The simulations presented used the $d t^{*}$ time step.

The expression of Talbot, Fig. 7a, is seen to over-predict the effect of the thermophoretic deposition by factors up to $60 \times$, exaggerating both the increase and decrease in deposition relative to isothermal conditions. It should be noted that a differing scale is required in this figure to plot the experimental data. Though extremely widely used to predict thermophoretic effects, this expression for the thermophoretic coefficient is not suitable for high particle-gas thermal conductivity ratios such as salt in air.

Simulations using the expression of Young, Fig. 7b showed results unrepresentative of the experimental values, including the presence of reversed thermophoresis. This is a process where particles move up the temperature gradient due to the thermal creep flow changing direction. Reversed thermophoresis has not been observed experimentally for the gas conditions simulated. It is concluded that this expression for $\Phi$ is not accurate for the computation of the thermophoretic force for either this $K n$ or $\Lambda$ regime. 
Simulations using the expression of Beresnev and Chernyak, Fig. 7c indicate close reproduction of the experimental trends. Both increasing and decreasing thermophoresis are well-predicted. In the increasing thermophoresis direction $\left(\overline{f_{d}}>1\right)$, simulated results are within $13 \%$ of the experimental. In the decreasing thermophoresis direction $\left(\overline{f_{d}}<1\right)$, simulated results are further from the experimental data: $\mathrm{T}_{D}$ simulation results were up to three times lower than the experimental. The $\mathrm{T}_{E}$ simulation results were significantly lower than the experimental, by up to two orders of magnitude. This expression for $\Phi$ is not widely used, due to the necessity to interpolate tabulated coefficients during the calculation process. Its improved performance for this particle material, and others noted in the literature, indicate that its use is beneficial.

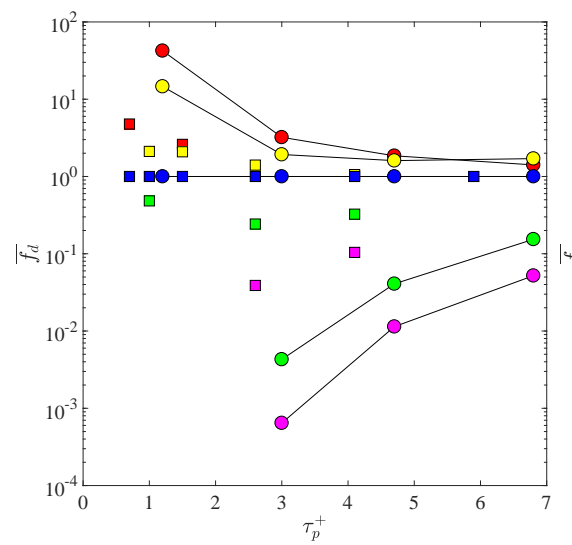

(a) $\Phi$ of Talbot

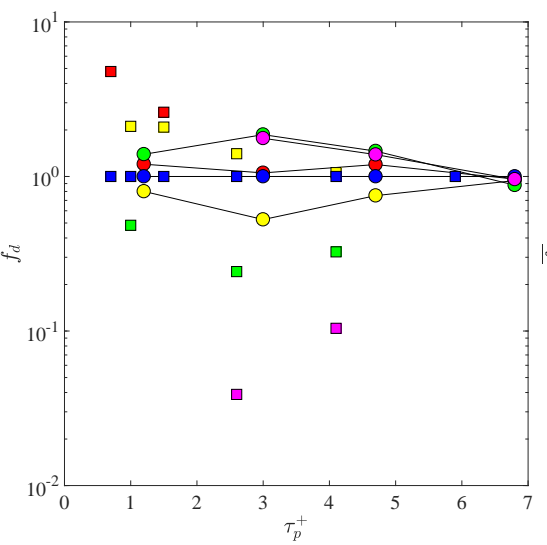

(b) $\Phi$ of Young

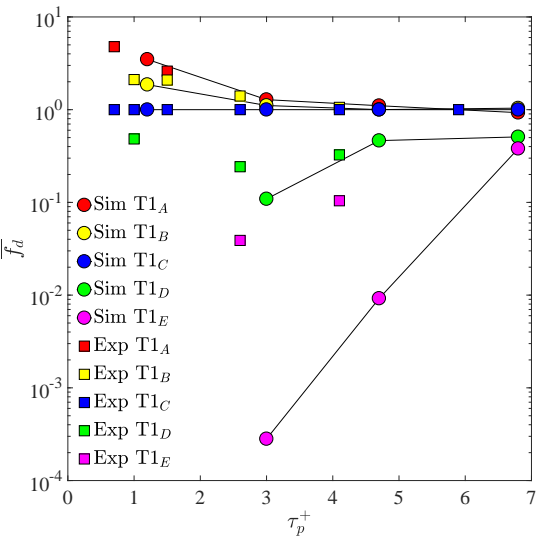

(c) $\Phi$ of Beresnev and Chernyak

Fig. 7 Normalised deposition fraction vs. $\tau_{p}^{+}$for thermophoretic tests. Experimental and varying expressions for thermophoretic coefficient $\Phi$. Note differing scale in plot Fig. $7 \mathbf{a}$.

\section{Conclusions}

Numerical simulations have been carried out of high temperature horizontal pipe flow particle deposition experiments. The continuous random walk model was used to generate fluid instantaneous turbulent fluctuations in order to capture the stochastic effects of turbulence when Lagrangian particle tracking was applied to a RANS continuous phase solution.

Simulations at ambient conditions indicated that the CRW as-is was not able to capture the effect of adding the gravitational force under horizontal flow conditions. Addition of the crossing trajectories effect, where a decorrelation of particle-gas velocities is introduced, was able to reduce this discrepancy from an order of magnitude to 0.9-49.7\%. However the simulations were not able to capture the reduction in $V_{d}^{+}$for $\tau_{p}^{+}>8$ seen experimentally, due to the imposed all-stick boundary condition.

When the high temperature experiments were simulated it was found that the expression of Talbot for the thermophoretic coefficient, whilst widely used, was not able to accurately predict the relative change in deposition rate from isothermal to thermophoretic conditions. The expression of Beresnev and Chernyak was found to closely predict these relative changes in deposition rate, and is proposed to be a more suitable method for the calculation of the thermophoretic force on particles in these regimes of Knudsen number and thermal conductivity ratio.

\section{References}

[1] Chambers, J., "The 1982 Encounter of British Airways 77 with the Mt. Galunggung Eruption Cloud. Paper no. 85-0097," 23rd Aerospace Sciences Meeting, Reno, Nevada, 1985.

[2] Clarkson, R., Majewicz, E., and Mack, P., "A re-evaluation of the 2010 quantitative understanding of the effects volcanic ash has on gas turbine engines," Proceedings of the IMechE Part G: Journal of Aerospace Engineering, Vol. 230, No. 12, 2016, pp. 2274-2291.

[3] Suman, A., Kurz, R., Aldi, N., Morini, M., Brun, K., Pinelli, M., and Spina, P., "Quantitative CFD Analyses of Particle Deposition on a Subsonic Axial Compressor Blade - Part I: Particle Zones Impact,” Journal of Turbomachinery, Vol. 137, No. 2, 2014, pp. 021009-021009-14. 
[4] Whitaker, S., Prenter, R., and Bons, J., “The Effect of Freestream Turbulence on Deposition for Nozzle Guide Vanes,” Journal of Turbomachinery, Vol. 137, No. 12, 2016, p. 121001.

[5] Wylie, S., Bucknell, A., Forsyth, P., McGilvray, M., and Gillespie, D., "Reduction in flow parameter resulting from volcanic ash deposition in engine-representative cooling passages," Journal of Turbomachinery, Vol. 139, No. 3, 2017, pp. 031008-13.

[6] Liu, B., and Agarwal, J., "Experimental observation of aerosol deposition in turbulent flow," Journal of Aerosol Science, Vol. 5, No. 2, 1974, pp. 145-155.

[7] Schiller, L., and Naumann, A., “A drag coefficient correlation,” Zeitschrift des Vereins Deutscher Ingenieure, Vol. 77, 1935, pp. 318-320.

[8] Brock, J., "On the theory of thermal forces acting on aerosol particles," Journal of Colloid Science, Vol. 17, 1962, pp. 768-780.

[9] Talbot, L., Cheng, R., Schefer, R., and Willis, D., “Thermophoresis of particles in a heated boundary layer," Journal of Fluid Mechanics, Vol. 101, No. 4, 1980, pp. 737-758.

[10] Beresnev, S., and Chernyak, V., "Thermophoresis of a spherical particle in a rarefied gas: Numerical analysis based on the model kinetic equations," Physics of Fluids, Vol. 7, No. 7, 1995, pp. 1743-1756.

[11] Young, J., "Thermophoresis of a Spherical Particle: Reassessment, Clarification, and New Analysis," Aerosol Science and Technology, Vol. 45, No. 8, 2011, pp. 927-948.

[12] Waldmann, L., "Uber die Kraft eines inhomogen Gases auf kleine suspendierte Kugeln.” Z. Naturforschung, Vol. 14, No. a, 1959, p. 589-599.

[13] Gosman, A., and Ionnides, E., "Aspects of Computer Simulation of Liquid-Fuelled Combustors," Journal of Energy, Vol. 7 , No. 6, 1983, pp. 482-490.

[14] Tian, L., and Ahmadi, G., "Particle deposition in turbulent duct flows - comparisons of different model predictions," Journal of Aerosol Science, Vol. 38, No. 4, 2006, pp. 377-397.

[15] Dehbi, A., "Turbulent particle dispersion in arbitrary wall-bounded geometries: A coupled CFD-Langevin-equation based approach," International Journal of Multiphase Flow, Vol. 34, No. 9, 2008, pp. 819-828.

[16] Ghahramani, E., Abouali, O., Emad, H., and Ahmadi, G., "Numerical analysis of stochastic dispersion of micro-particles in turbulent flows in a realistic model of human nasal/upper airway," Journal of Aerosol Science, Vol. 67, No. 1, 2014, pp. $188-206$.

[17] Dehbi, A., and de Crechy, F., "Validation of the Langevin particle dispersion model against experiments on turbulent mixing in a T-junction," Powder Technology, Vol. 206, No. 3, 2011, pp. 312-321.

[18] Sippola, M., and Nazaroff, W., "Experiments Measuring Particle Deposition from Fully Developed Turbulent Flow in Ventilation Ducts," Aerosol Science and Technology, Vol. 38, No. 9, 2004, pp. 914-925.

[19] Montgomery, T., and Corn, M., "Aerosol deposition in a pipe with turbulent airflow," Aerosol Science, Vol. 1, No. 3, 1970, pp. $185-213$.

[20] Forsyth, P., Gillespie, D., and McGilvray, M., "Experimental deposition of $\mathrm{NaCl}$ particles from turbulent flows at gas turbine temperatures. Paper no. 160," ISROMAC, 2017.

[21] Kvasnak, W., Ahamadi, G., Bayer, R., and Gaynes, M., "Experimental investigation of dust particle deposition in a turbulent channel flow," Journal of Aerosol Science, Vol. 34, No. 6, 1993, pp. 795-815.

[22] Hurley, M., "SFPR Handbook of Fire Protection Engineering," Springer-Verlag New York, ISBN 978-1-4939-2564-3, 2008.

[23] Hilsenrath, J., "Tables of thermal properties of gases : comprising tables of thermodynamic and transport properties of air, argon, carbon dioxide, carbon monoxide, hydrogen, nitrogen, oxygen, and steam," U.S. Dept. of Commerce, National Bureau of Standards, Washington D.C., 1955.

[24] Iliopoulos, I., Mito, Y., and Hanratty, T., "A stochastic model for solid particle dispersion in a nonhomogeneous turbulent field," International Journal of Multiphase Flow, Vol. 29, No. 3, 2003, pp. 375-394.

[25] Bocksell, T., and Loth, E., "Random Walk Models for Particle Diffusion in Free-Shear Flows,” AIAA Journal, Vol. 39, No. 6, 2001, pp. 1086-1096. 
[26] Forsyth, P., Gillespie, D., and McGilvray, M., "Validation and Assessment of the Continuous Random Walk Model for Particle Deposition in Gas Turbine Engines, GT2016-57332," ASME Turbo Expo, 2016.

[27] Yudine, M., "Physical considerations on heavy-particle diffusion," Advances in Geophysics, Vol. 6, No. 1, 1959, pp. $185-191$.

[28] Arcen, B., Taniere, A., and Oesterle, B., "Influence of the gravity field on the turbulence seen by heavy discrete particles in an inhomogeneous flow," ERCOFTAC International Symposium on Engineering Turbulence and Measurements - ETMM6, 2005, pp. 949-958.

[29] Kallio, G., and Reeks, M., "A numerical simulation of particle deposition in turbulent boundary layers," International Journal of Multiphase Flow, Vol. 15, No. 3, 1989, pp. 433-446.

[30] Csanady, G., "Turbulent Diffusion of Heavy Particles in the Atmosphere," Journal of Atmospheric Sciences, Vol. 20, No. 5, 1963, pp. 201-208. 